\title{
Asociación entre dolor crónico, deterioro cognitivo y demencia en una cohorte longitudinal de adultos mayores
}

\author{
Association Between Persistent Pain and Memory Decline and Dementia in a Longitudinal Cohort of Elders
}

\section{Comentado de:}

Whitlock, E. et al. Association Between Persistent Pain and Memory Decline and Dementia in a Longitudinal Cohort of Elders. JAMA, 2017. 177(8):1146-1153. PMID: 28586818 ${ }^{1}$

\section{Objetivo}

Determinar la asociación entre el dolor crónico y el desarrollo de deterioro cognitivo y demencia en pacientes mayores.

\section{Diseño, lugar y pacientes}

Estudio de cohorte realizado en EE.UU. que incluyó entrevistas bianuales a adultos con edad de 62 años o más $(\mathrm{N}=10.065)$. La información fue extraída a partir de la base de datos de la encuesta Health and Retirement que incluía preguntas sobre aspectos del dolor y de la presencia de deterioro cognitivo entre los años 1998 y 2000 . El análisis de datos se realizó entre Junio y Octubre del 2016.

\section{Medición de resultados principales}

Los desenlaces principales fueron la puntuación de memoria compuesta y la probabilidad de desarrollar demencia, estimadas mediante la combinación de resultados de pruebas neuropsicológicas y entrevistas con informantes, realizadas entre 2000 y 2012. Se utilizaron modelos lineales de efectos mixtos para estimar la asociación entre dolor crónico con evaluación de la tendencia cognitiva posterior, ajustado por características demográficas y comorbilidades, relevadas en el año 2000.

La hipótesis fue que el dolor persistente (definido como aquel presente cuando el participante informaba problemas con dolor moderado o severo con frecuencia) predeciría una disminución acelerada de la memoria y una mayor probabilidad de desarrollar demencia. Para cuantificar el impacto del dolor persistente en la independencia funcional, se combinaron los resultados primarios con información sobre la asociación entre la memoria y la capacidad de administrar medicamentos.

\section{Resultados}

Al inicio del estudio, el dolor persistente afectaba al 10,9\% de los participantes y se asoció con mayores síntomas depresivos y limitaciones en las actividades de la vida diaria. Después del ajuste por covariables, el padecimiento de dolor persistente se asoció con un deterioro 9,2\% más rápido de la memoria (Intervalo de Confianza [IC] del 95\%: 2,8\% a 15\%) en comparación con los pacientes sin dolor persistente. Después de 10 años, esta disminución acelerada de la memoria implicaba un 15,9\% más de riesgo relativo de incapacidad para administrar medicamentos y un $11,8 \%$ más de riesgo relativo de incapacidad para administrar las finanzas de forma independiente.

La probabilidad ajustada de desarrollar demencia aumentó $7,7 \%$ más rápido (IC $95 \%: 0,55 \%$ a $14,2 \%$ ) en el grupo de dolor persistente, después de 10 años; esto se traduce en un aumento absoluto de $2,2 \%$ en la probabilidad dedesarrollar demencia en este grupo de pacientes.

Fuente de financiamiento: Este estudio fue financiado por una beca de entrenamiento del National Institute of General Medical Sciences, The National Institute on Aging, y The American Federation for Aging Research.

\section{Comentario}

En Argentina, la prevalencia estimada de pacientes con demencia es de 12,2\%, lo que representa aproximadamente 600.000 personas. Esta problemática no solo afecta al paciente respecto del requerimiento de cuidados, sino que además representa un desafío para su familia y cuidadores principales, y tiene un gran impacto en la Salud Pública (en términos de tratamientos, consultas, internaciones, insumos, medicaciones, etc) ${ }^{2}$.

Entre 50 y $80 \%$ de las personas mayores de 65 años presentan dolor por distintas causas. El dolor se asocia a diferentes enfermedades crónicas que aumentan su prevalencia con la edad. Como consecuencia, los adultos mayores presentan un deterioro en su funcionalidad, que puede asociarse a cuadros de ansiedad y depresión 2,3 .

\section{Conclusiones de la comentadora}

El estudio resumido documenta una asociación entre el dolor crónico y el deterioro cognitivo, aunque no quede claro si el tratamiento adecuado del síntoma disminuye el riesgo de desarrollar demencia. Como médicos de atención primaria debemos optimizar el tratamiento del dolor para disminuir el sufrimiento de los pacientes y mejorar su calidad de vida.

Micaela Alurralde [ Servicio de Medicina Familiar y Comunitaria, Hospital Italiano de Buenos Aires micaela.alurralde@hospitalitaliano.org.ar ]

Alurralde M. Asociación entre dolor crónico, deterioro cognitivo y demencia en una cohorte longitudinal de adultos mayores. Evid Actual Pract Ambul. 2019;22(3):e001080. Comentado de: Whitlock EL, Diaz-Ramirez LG, Glymour MM, Boscardin WJ, Covinsky KE, Smith AK. Association Between Persistent Pain and Memory Decline and Dementia in a Longitudinal Cohort of Elders. JAMA Intern Med. 2017;177(8):1146-1153. PMID: 28586818

\section{Referencias}

1. Whitlock E, Diaz-Ramirez G, Glymour M, et al. Association Between Persistent Pain and Memory Decline and Dementia in a Longitudinal Cohort of Elders. JAMA. 2017;177(8):1146 -1153. Publicacion Online: 5 de Junio de. Available from: 10.1001/jamainternmed.2017.1622.

2. and RGM. Enfermedad De Alzheimer. Clínica, tratamiento y rehabilitación. and others, editor. Barcelona: Editorial Masson; 2001.

3. Franco ML, Seoane-De-Lucas A. Characteristics of chronic pain among the elderly: management. Rev Soc Esp Dolor. 2001;8:29-38. 